
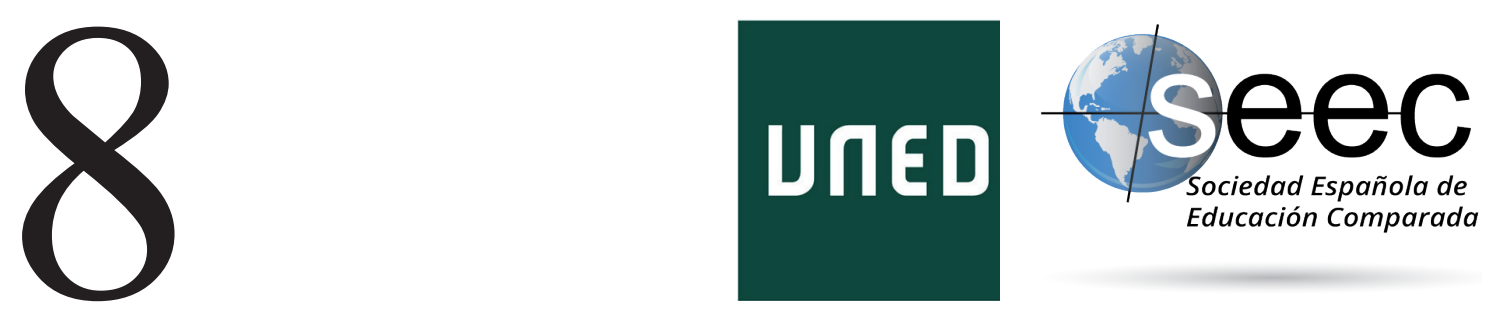

\title{
La autopercepción de la competencia digital en los docentes: variaciones tras el confinamiento
}

Self-perception of digital competence in teachers: variations after confinement

\section{Juan Tomás Asenjo Gómez*; Felix Asenjo Gómez ${ }^{* *}$}

DoI: $10.5944 /$ reec.38.2021.29032

\author{
Recibido: 3 de diciembre de 2020 \\ Aceptado: 10 de marzo de 2021
}

\footnotetext{
* Juan Tomás Asenso Gómez: Doctor en Ciencias de la Educación por la Universidad Pontificia Comillas. Profesor colaborador en los grados de Educación Infantil y Primaria y en el Máster de Secundaria en la Universidad Pontificia Comillas, así como en otras entidades universitaria: Universidad Escuni; Universidad Nebrija. Miembro del Grupo de Investigación sobre Políticas Educativas Supranacionales de la UAM. Datos de contacto: E-mail: tomas_asenjo@hotmail.com.

** Felix Asenjo Gómez: Licenciado en Filosofía y Letras, en la Sección de Ciencias de la Educación por la Universidad Pontificia Comillas. Diploma de Estudios Avanzados de la UNED. Profesor titular de las asignaturas de Teoría de la Educación e Historia y Corrientes Internacionales de la Educación en la Escuela de Magisterio ESCUNI. Datos de contacto: E-mail: fasenjo@escuni.es.
} 


\title{
Resumen
}

El año 2020 será recordado como el año en el que se vivió una tragedia humana, sanitaria, social y económica como consecuencia del virus COVID-19. La educación se enfrentó a un escenario no vivido con anterioridad y al igual que el resto de la sociedad se vio abocado a un severo confinamiento. Al confinamiento le siguió una reincorporación parcial del alumnado y en ambos escenarios la tecnología digital desempeñó un papel fundamental. La labor formativa se vio abocada a adoptar metodologías digitales con la urgencia que requería la situación y el profesorado tuvo que adaptarse sobre la marcha. Bimodalidad, educación sincrónica, clases online son ahora términos habituales que acompañan la compleja labor docente. El estudio que presentamos analiza el punto de partida del profesorado español y europeo respecto a las actitudes y niveles formativos en TIC, así como las posibles variaciones tras la irrupción de la pandemia. Para ello se han comparado los datos recogidos en el Estudio Internacional de Enseñanza y Aprendizaje, TALIS 2018, respecto al uso de las Tecnologías de la Información y la Comunicación, junto a los datos que arroja el Plan de Acción de Educación Digital 2021-2027 por medio de la Consulta Pública Abierta. Se puede concluir que si bien en los años previos al COVID-19 el profesorado presentaba unos altos niveles participativos de formación en TIC, sin embargo no valoraban la necesidad formativa con el mismo reconocimiento como una vez iniciada la pandemia. Aun así, planteamos la necesidad de un enfoque pedagógico en la formación digital que supere los aprendizajes técnicos proponiendo unas estrategias didácticas que permitan la equidistancia entre la formación presencial y la virtual.

\section{Palabras clave: Pandemia; Pedagogía; Digital; Holístico; Competencias}

\begin{abstract}
The year 2020 will be remembered as the year in which a human, health, social and economic tragedy was experienced as a result of the COVID-19 virus. Education faced a scenario not experienced before and like the rest of society it was doomed to severe confinement. The confinement was followed by a partial reincorporation of the students and in both scenarios digital technology played a fundamental role. The formative work was forced to adopt digital methodologies with the urgency that the situation required and the teaching staff had to adapt as they go. Bimodality, synchronous education, online classes are now common terms that accompany the complex teaching work. The study that we present analyzes the starting point of Spanish and European teachers regarding attitudes and training levels in ICT, as well as the possible variations after the outbreak of the pandemic. For this, the data collected in the Teaching And Learning International Study, TALIS 2018, regarding the use of Information and Communication Technologies, have been compared, together with the data provided by the Digital Education Action Plan 2021-2027 by through the Open Public Consultation. It can be concluded that although the teachers, before COVID-19 crisis, had high participation levels on ICT training, they did not value the need to be trained with the same recognition as once the pandemic began. Even so, we propose the need for a pedagogical approach in digital training that goes beyond technical learning by proposing didactic strategies that allow the equidistance between face-to-face and virtual training.
\end{abstract}

Keywords: Pandemic; Pedagogy; Digital; Holistic; Skills. 


\section{Introducción}

El término «cambio», al que se recurre con insistencia para tratar de interpretar los fenómenos que acompañan a la sociedad actual, nos habla de mudanza, fugacidad, eventualidad, transformación, alteración... Para algunos autores gran parte de las transformaciones que estamos viviendo tiene su origen en las nuevas tecnologías (Schwab y Davis, 2018); para otros, es producto de una modernidad mal gestionada (Bauman, 2008) y de una postmodernidad carente de valores (Ruiz, 2010); también nos encontramos con quienes piensan que son ciclos históricos que se deben interpretar e incluso quien habla de innovaciones disruptivas (García Aretio, 2019). Centrados en el área educativa, Mora (2011) concretaba las reformas que se vienen produciendo, con mayor o menor intensidad: la sociedad global; la universalidad de la educación superior; la sociedad del conocimiento; internet y sus derivados. Si bien los tres primeros han ido ocasionando un ajuste progresivo de los paradigmas educativos, la tecnología digital ha significado toda una revolución, entendida como un cambio profundo en las estructuras académicas y en la propia sociedad. Las tecnologías proyectan toda una novedosa oferta metodológica paralela a la denominada, y considerada, «metodología tradicional», contando con el sólido respaldo que imprimen las plataformas sociales, lo intuitivo de su manejo y la velocidad en el procesamiento de datos. Esta irrupción exponencial de las tecnologías en el entorno académico ha supuesto una transformación estructural del modelo de enseñanza y aprendizaje, evidencia que se puede constatar en la proliferación de los cursos formativos para docentes y en su mayor presencia en las aulas.

La evolución digital mantiene un ritmo de crecimiento con altos niveles de conectividad entre los usuarios; sin embargo, esta auténtica renovación tiene su origen a mediados del siglo pasado, con la denominada Tercera Revolución Industrial o Revolución científicotecnológica, definida por una tecnología digital que daría lugar a la era de la información. Entonces, ¿̇por qué seguir denominando nuevas tecnologías a una realidad que nos acompaña de manera global desde finales del siglo XX? Las tecnologías ya hace tiempo que dejaron de ser nuevas para pasar a estar plenamente integradas en nuestra era.

Más allá de la revisión terminológica, el contexto digital que domina el siglo XXI demanda una educación capaz de entender y atender las nuevas exigencias, para así facilitar las competencias personales que se necesitan en el renovado entramado social. Una nueva noción de aprendizaje, tanto en lo referente a las formas como a los contenidos, implica nuevos modelos formativos que de manera automática han sido reclamados a las instituciones educativas y que, por extensión, ha puesto el foco de atención en el profesorado. En torno a todo este discurso Ribes (2011) apunta un dato que influye en la tan buscada calidad de los centros y que pasa por una formación del profesorado en competencias, pues sobre éstas va a desarrollar su actividad: "para aprender una competencia se requiere de alguien que la domine previamente y que el aprendizaje tenga lugar en la situación en que se ejercita en la forma de conocimiento funcional (y no puramente formal o declarativo)» (p. 42). Una vez que las competencias están presentes en lo macro, discursos pedagógicos, y en lo micro, programaciones, es inevitable seguir formando y apoyando al profesorado. Aspecto este que invariablemente se menciona, pero sobre el cual pocas veces se actúa con determinación (Egido, 2011).

La previsión de este inexorable proceso se venía anunciando desde las organizaciones internacionales. La OCDE (1989) ya hacía hincapié en la necesidad de un profesorado formado en las nuevas tecnologías y en su aplicación en el aula. La UNESCO (1999) 
relacionaba a los docentes con las nuevas tecnologías y los nuevos procesos de enseñanza. Años más tarde, la propia UNESCO (2008) instaba a definir los estándares de competencia en TIC de los profesores. Por su parte, desde el Consejo Europeo (DO, L394/10, 2006) se ha afianzado la formación desde las competencias con la Recomendación sobre las competencias clave para el aprendizaje permanente. Una apuesta que pretende dotar al ciudadano de unas estrategias acordes a una sociedad en continua evolución. Postura que se ha revalidado en la Recomendación del Consejo (DO, C189/1, 2018): las competencias clave son aquellas que todas las personas precisan para su realización y desarrollo personales, su empleabilidad, integración social, estilo de vida sostenible, éxito en la vida en sociedades pacíficas, modo de vida saludable y ciudadanía activa. Si analizamos la evolución en la denominación de las diferentes competencias, observamos que la única que se mantiene sin variación es la competencia digital. Si bajamos al detalle de su definición (Tabla 1) nos encontramos con una lógica evolución transcurridos 12 años.

Tabla 1.

Las competencias clave

\begin{tabular}{lll}
\hline & Resolución 2006 & Resolución 2018 \\
\hline Denominación & Competencia digital & Competencia digital
\end{tabular}

Meta Un acceso seguro a las tecnologías de la sociedad de la información (TSI).

Incluye

Manejar herramientas para la comprensión de la información y para el uso de los servicios localizados en Internet.

La utilización de los ordenadores en el uso de información y comunicación, y en el acceso a Internet.

Requerimiento Una actitud crítica y reflexiva; un uso responsable y participativo con la comunidad.
La responsabilidad en el uso de las tecnologías digitales para el aprendizaje.

La alfabetización de información y mediática.

Fomentar una ciudadanía activa, inclusiva y colaboradora.

Una actitud reflexiva y crítica; actitudes éticas.

Fuente: elaboración propia a partir de DO, L394/10, 2006; DO, C189/1, 2018

Se puede observar cómo se pasa de hablar de las tecnologías de la sociedad de la información a las tecnologías digitales centradas en el aprendizaje. Aparece la alfabetización mediática, más allá de la participación en redes. En lo referente a la capacitación, el interés ha pasado del uso de herramientas al apoyo a una ciudadanía activa teniendo presente la inclusión social. Como requisito, el uso responsable se ha traducido en la necesidad de unos planteamientos éticos.

La perspectiva ética es obligada en la era de la revolución digital, pues los cambios están anticipándose a las teorías, la práctica a la reflexión y la acción a la moral. Lo que se 
valora es ir alcanzando objetivos inmediatos, pero estancos respecto a grandes proyectos. Este mismo esquema se ha trasladado a las relaciones sociales, donde la individualidad del ordenador o de la telefonía pretende convertirse en el estilo de interacción, por paradójico que resulte. Este proceder ha alcanzado a los modelos educativos, favoreciendo la especificidad de los conocimientos de forma contraria a los principios holísticos de una educación integral. Siendo esto así, el hecho de dejarse atrapar por la automatización de una formación técnica, utilitarista, nos dirigiría hacia el reduccionismo de un saber hacer economicista. Es por ello que la dimensionalidad de las competencias no puede dejarse polarizar por el desempeño, pues el conocimiento, con su base histórica nos acerca a lo que somos y las actitudes nos recuerdan quiénes somos y con quién convivimos.

La ciudadanía está inmersa en contextos cada vez más complejos, por lo novedoso, por las fluctuaciones sociales, por la internacionalización, lo que conllevará de forma ineludible a la construcción de una sólida estructura personal que facilite el análisis de las bases informativas, que no formativas. Esta base personal, y una actitud reflexiva, movilizarán el proceso de búsqueda, análisis y asimilación de información como camino de actuación responsable ante las diferentes necesidades. Con todo, para proceder a una primera aproximación a la información, la tecnología se ha presentado como un recurso esencial; no contar con el acceso a la tecnología o la falta de recursos personales para su utilización nos conducen a la denominada brecha digital, que en su forma más severa se relaciona con pobreza y exclusión social (Olarte, 2017). Si bien los datos que se manejan en el contexto supranacional de la Unión Europa respecto al acceso a internet en los hogares (Tabla 2) muestran una constante progresión en los últimos años; alcanzándose cuotas cercanas al cien por cien en algunos países. También muestra lo desigual del acceso al principio de la década; y se mantienen datos no tan optimistas con respecto a algunos países comunitarios como Grecia (71 \%) o Bulgaria (67\%).

Tabla 2.

Acceso a internet hogares.

\begin{tabular}{lcc}
\hline & 2012 & 2017 \\
\hline EU-28 & 76 & 87 \\
\hline Netherlands & 94 & 98 \\
\hline Denmark & 92 & 97 \\
\hline Luxembourg & 93 & 97 \\
\hline Finland & 87 & 94 \\
\hline Germany & 85 & 93 \\
\hline Austria & 79 & 89 \\
\hline Ireland & 81 & 88 \\
\hline Belgium & 78 & 86 \\
\hline France & 80 & 86 \\
\hline Malta & 77 & 85 \\
\hline Czech Republic & 65 & 83 \\
\hline Spain & 67 & 83 \\
\hline
\end{tabular}

Fuente: Eurostat 
No obstante, en el trabajo de Lago (2019) se exponen tres dimensiones en el concepto de brecha digital que van más allá de los niveles de acceso a internet: la brecha de acceso a internet existente entre los países industrializados y en los países en vías de desarrollo; la brecha social, quienes acceden a la información frente a los denominados informacionalmente pobres; y la brecha democrática, que impide participar en la vida pública.

Junto a la preocupación por las consecuencias de una brecha digital no resuelta, se mantiene en el contexto europeo otra de las preocupaciones presente en las propias competencias clave, la inclusión. Las TIC facilitan el acceso a la información, la mejora social y laboral, pero no hay que olvidar el papel de la tecnología como herramienta de inclusión (Pegalajar, 2017; Cabero y Valencia, 2019), de poner a las TIC al servicio de la inclusión educativa (Rodríguez y Arroyo, 2014). La propia Comisión Europea (2020a) subraya que si los educadores realizan un uso eficaz de la tecnología digital contribuyen a una educación inclusiva y de calidad.

\section{El colegio se muda a los hogares. Confinamiento}

El año 2020 pasará a la historia como el año de la tragedia humana, sanitaria, social y económica derivado por la pandemia COVID-19. A nivel educativo, el mes de marzo del 2020 supuso un escenario desconocido que se tradujo en el cierre de los centros y en el inicio de la formación online. Miles de alumnos se vieron confinados en sus casas en medio del curso académico, con la incertidumbre de cómo sería su formación y su evaluación a partir de ese momento. El paso siguiente fue la reorganización de la actividad académica aupando a la tecnología como medio indispensable y como fuente de no pocos problemas: escasez de recursos, interrogantes sobre la confidencialidad, dificultades en el uso de las aplicaciones. Junto a la desigualdad que pronto se hizo patente entre aquellos alumnos que no tenían los medios ni la posibilidad de usarlos frente a los que sí, el amplio espacio de tiempo durante el cual las aulas se mantuvieron cerradas reveló el frágil equilibrio en el que se encontraban los diferentes agentes educativos: autoridades, instituciones, familias y docentes, condicionando cualquier toma de decisiones. Sin embargo, donde más se fijó el discurso social fue en el profesorado, al que se atribuyó unas carencias digitales, así como unas actitudes reacias a la hora de afrontar a una modalidad tan diferente al formato presencial. La encrucijada a la que se enfrentaba toda la comunidad educativa implicaba responder de manera urgente a las nuevas exigencias tecnológicas, pero sin renunciar a la calidad docente tanto en contenidos como en evaluaciones. De un día para otro el debate sobre la formación en tecnologías digitales que Core (2012) presentaba como resistir o innovar quedó aparcado ante lo acuciante de la situación.

La figura del profesor adquiría así una nueva relevancia. Si hablamos del perfil del docente quizá sea más adecuado hablar del perfil competencial del docente. Esta leve pero decisiva distinción entre cualidades y perfil competencial pone en evidencia la importancia adquirida no tanto por las características del profesor como por las necesidades que presenta el grupo y las materias. El profesor se muestra, al tiempo que en transmisor de conocimientos, como un canalizador de la información, de esa ingente información asociada a las TIC y que rodea al alumno. Trabajar, por tanto, en la formación de esa competencia digital en el profesorado adquiere toda la pertinencia que marcan los acontecimientos (Ribés, Moliner y Alegre, 2020). 
La cuestión estriba en si la tecnología se posiciona como un complemento de la educación (Fernández Prieto, 2015; Tobón, 2020), viene a implementarla (Tumino y Bournissen, 2019) o va a establecerse como decisiva (Torrecillas, 2020). Lo que parece indudable es que hace tiempo que el entorno digital superó el periodo de integración en los entornos educativos para dar lugar a nuevas estrategias de enseñanza y de aprendizaje, quedando como tema pendiente superar la distancia que se crea entre el alumnado en el modelo no presencial (Sangrà, 2020).

La COVID-19 ha acelerado todo el proceso reformador en lo referente al binomio educación y tecnología, centrando la atención en la revisión de la formación inicial y continua del profesorado. Respecto a la formación continua, se ha trabajado sobre la marcha para facilitar los escenarios de enseñanza online y bimodal, con los que se ha buscado hacer frente a la no presencialidad del alumnado. Pero la inercia nos debe conducir hacia una renovada planificación de los programas formativos del profesorado; programas habilitantes para un profesorado que se va a encontrar con un contexto educativo digital y online. Así, la competencia digital queda unida al desarrollo profesional docente desde el momento en el que las tecnologías forma parte de nuestro entorno laboral y social, por lo que se puede hablar de una profesionalización docente en el área de las TIC (Tejada y Pozos, 2018). Se trata de una competencia, la digital, equiparable a lo que en su momento supusieron los planes de alfabetización (Fernández Enguita, 2020).

\section{Y ahora ¿́cómo seguimos? Postconfinamiento}

En 1995 la UNESCO ya abordaba la necesidad de renovar los modelos de enseñanza y aprendizaje por medio de métodos pedagógicos que tuvieran en cuenta las tecnologías de la información y la comunicación. Entonces, cómo es posible que las competencias digitales rivalicen o estén directamente ubicadas en un prisma diferente a las competencias pedagógicas. Desde el informe Cedefop (2020) se han delineado los retos que afronta el personal docente en esa transición de la presencialidad a los espacios de aprendizaje virtual (Tabla 3). Retos que están teniendo un desigual tratamiento.

Tabla 3 .

Retos del personal docente en la formación virtual

Desafíos que enfrenta el personal docente

Ámbitos de actuación

-Ausencia de acceso a equipos y conexión a

internet para ofrecer aprendizaje a distancia

-Ámbito económico: partidas

presupuestarias

- Falta de habilidades y competencias digitales para hacer un uso eficiente de las plataformas

-Ámbito instrumental:

- Escasa experiencia en la creación de contenidos de enseñanza digital formación técnica

- Falta de experiencia en aprendizaje digital y otras pedagogías efectivas de aprendizaje a distancia

-Ámbito pedagógico: formación adaptada y contextualizada

-Ámbito didáctico: formación en sombra y tutorizada

- Preocupaciones sobre asuntos de privacidad, -Ámbito deontológico: aspectos éticos derechos de autor y protección de datos

Fuente: adaptado a partir de Cedefop, 2020. 
La inversión económica progresiva y constante (datos de acceso a internet) y la formación en el uso de las herramientas digitales (niveles formativos de los docentes) están fuera de toda duda y han ido cumpliendo diferentes fases de la denominada alfabetización digital. El ámbito ético también ha ocupado espacios de reflexión relacionados con la protección de datos y la privacidad. Pero la necesidad de atender de manera urgente una docencia en línea ha mostrado déficits que no se consideraron trabajar en su momento. Se intensificaron los esfuerzos en las aspectos digitales técnicos, en el manejo de las numerosas aplicaciones, pero se realizó un menor hincapié en las que denominamos competencias pedagógicas, es decir, los conocimientos, destrezas y actitudes que presenta el profesorado para hacer que los estudiantes aprendan en entornos digitales.

Desde las instituciones europeas se estableció el Marco Europeo para la competencia digital del profesorado (DigCompEdu) (Comisión Europea, 2017), dentro del Marco Común de Competencia Digital Docente, en coherencia con esa correlación entre alfabetización, competencias y desarrollo del profesorado. El DigCompEdu está dirigido a los educadores de todos los niveles de la educación y propone seis áreas de competencias diferentes (generando un total de 22 competencias) donde se considera a las áreas 2 a 5 su núcleo pedagógico:

- Área 1. Compromiso profesional: se ocupa de los aspectos profesionales.

- Área 2. Recursos digitales: tanto su creación como su distribución .

- Área 3. Pedagogía Digital: busca coordinar las aplicaciones digitales con los procesos educativos.

- Área 4. Evaluación y retroalimenación: optimizer los procesos evaluativos.

- Área 5. Empoderar a los estudiantes.

- Área 6. Facilitar la competencia digital de los estudiantes.

DigCompEdu analiza los nuevos métodos de formación, la interacción con los estudiantes, el trabajo colaborativo y el apoyo al trabajo autónomo. Pero, durante la pandemia lo que se ha echado en falta ha sido una didáctica equiparable a la realizada en un aula física, esa interacción con los alumnos, por lo que lo que los esfuerzos se deben concentrar en una pedagogía digital materializada en propuestas concretas que faciliten esa proximidad limitada por la distancia. Sanabria y Cepeda (2016) establecen la siguiente graduación de la competencia digital: alfabetización, sabiduría, identidad y ciudadanía digital. El nivel de identidad incorpora esa dimensión creativa y comunicativa que permitirá al alumno avanzar en su desarrollo personal más allá de los escenarios de modalidad presencial o virtual.

Formarse en didácticas digitales tendrá que ocupar, a nuestro juicio, un espacio estratégico en los programas de formación de formadores, atendiendo a una triple finalidad:

- como metodología que equipare la no presencialidad a la presencialidad, representando esa equidistancia un principio de ética profesional;

- como elemento clave en la formación digital del alumnado, capacitándolo para una ciudadanía plena y solidaria;

- como garantía de una educación holística, atendiendo la individualidad de cada persona, pues todo alumno es diverso, presenta multitud de facetas y vive en comunidad. 


\section{El profesorado en la coyuntura pre y post confinamiento}

El estudio que se presenta examina los datos recogidos en el Estudio Internacional de Enseñanza y Aprendizaje, TALIS 2018, informe español (MEFP, 2019) respecto al uso de las Tecnologías de la Información y la Comunicación, junto a los datos que arroja el Plan de Acción de Educación Digital 2021-2027 por medio de la Consulta Pública Abierta (OPC, por sus siglas en inglés) que ha llevado a cabo la Comisión Europea (2020b). A través del análisis realizado pretendemos determinar el punto de partida del profesorado español y europeo respecto a las actitudes y niveles formativos en TIC y valorar si se ha producido una variación en las mismas tras la irrupción de la pandemia.

En segundo lugar se aborda la percepción, en clave de ventajas e incovenientes, que los docentes tienen respecto de una enseñanza online o semipresencial, así como la influencia que la brecha digital tendría en la misma.

El Estudio Internacional de Enseñanza y Aprendizaje presentaba los datos recogidos tras la aplicación de un cuestionario que se cumplimentó por aquellos docentes que habían participado en al menos alguna actividad de desarrollo profesional. Se les ofreció una lista con 14 temas, entre los que se encontraba Destrezas de las Tecnologías de la Información y la Comunicación aplicadas a la enseñanza. Sobre estos temas tenían que señalar el número de participaciones, así como el nivel o necesidad de formación con la siguiente graduación: «ninguna necesidad», «escasa necesidad», «necesidad media», «una gran necesidad». El total de la muestra para el estudio del año 2018 contó con 4.00o docentes de Primaria y Secundaria distribuidos entre 200 centros educativos y 20 docentes por centro, tanto de la Unión Europea como de países miembros de la OCDE. Los resultados son los siguientes:

a) Educación Primaria: En el caso español, el profesorado de Educación Primaria que ha participado en diversas actividades de desarrollo profesional se encuentra en la horquilla de $55 \%$ y $65 \%$, ocupando las destrezas en TIC un $63 \%$. Respecto a la necesidad de formación que sostienen el profesorado en el rango de «gran necesidad», las destrezas TIC ocupan un $22 \%$. Por comunidades, los datos son muy parejos, con un $24 \%$ en Madrid, un 20 \% en Cantabria, La Rioja o Cataluña, un 18 \% en Castilla y León y un 16 \% en Asturias.

En cuanto a las actividades formativas de desarrollo profesional, las destrezas TIC, junto con la participación en formación en las áreas de gestión del aula (59 \%) y en destrezas transversales (59\%), son las más solicitadas. Con menor demanda se encuentra la formación sobre el análisis y uso de las evaluaciones del alumno (50 \%), enfoques de aprendizajes individualizados (50 \%). Por debajo quedan la cooperación entre familias y docentes (43\%) o la comunicación con personas de diferentes culturas y países (25\%).

b) Educación Secundaria. Los niveles de participación muestran unos datos también altos, entre el $40 \%$ y el $60 \%$, donde el área de contenidos formativos en destrezas TIC aplicadas a esta etapa alcanza un $68 \%$ en España frente al $61 \%$ promedio de la OCDE y al total de la UE con un $59 \%$. Los docentes de secundaria subrayan como «gran necesidad» de formación en las TIC en algo menos de un 16 \% en España, ligeramente inferior que sus compañeros de Primaria, y también por debajo de la UE (18 \%) y de la OCDE (19\%). Por comunidades se mantienen los datos de forma muy similar, Madrid con un $19 \%$, La Rioja (18 \%), Cataluña (17 \%) y Castilla y León (15 \%). Frente a otras áreas, la formación en TIC es en la que los docentes más participan, por delante tanto a nivel nacional 
como internacional, ocupando los últimos puestos en la participación la enseñanza en un entorno multicultural y plurilingüe (22 \% OCDE, $20 \%$ UE) y en comunicación con personas de diferentes culturas o países (19 \% OCDE, 18 \% UE); en España los niveles participativos son algo más elevados, con un $32 \%$ y un $23 \%$ respectivamente en estos dos campos.

Si valoramos la evolución de los datos españoles en el período que ocupa del año 2013 al año 2018 se muestra una falta de progresión e incluso un retroceso en la demanda de alta necesidad de formación respecto a las destrezas TIC (Tabla 4).

Tabla 4 .

Evolución porcentual necesidades en formación en TIC.

\begin{tabular}{lcc}
\hline Año & $\mathbf{2 0 1 3}$ & $\mathbf{2 0 1 8}$ \\
\hline España & 14 & 15 \\
\hline OCDE & 19,1 & 17,7 \\
\hline
\end{tabular}

Fuente: Talis, 2018

La Consulta Pública Abierta (Comisión Europea, 2020b) recogió datos desde el 18 de junio de 2020 hasta el 4 de septiembre de 2020, recibiendo un total de 2.716 respuestas desde 60 países. Las numerosas respuestas recibidas desde Rumanía (58 \%) generaron dos análisis paralelos: «todos los encuestados» y «todos los encuestados menos Rumanía». Para nuestra investigación vamos a utilizar las 1.140 respuestas de la encuesta sin los datos de Rumanía, de los que 130 corresponden a España. El perfil de los encuestados engloba instituciones de educación, organizaciones, proveedores de tecnología digital y las respuestas a título individual (69,8 \%), donde están ampliamente representados padres y educadores. La consulta incluía cuatro bloques temáticos, dedicando el tercero de los bloques a recoger información sobre la visión digital en Europa por parte de los participantes.

Dentro de los diferentes aspectos abordados se incluyó: a) puntos de vista sobre las competencias digitales; b) puntos de vista sobre el papel de la educación digital en el futuro; c) puntos de vista sobre cómo la Unión Europea podría agregar valor cuando se trata de educación digital.

a) Puntos de vista sobre las competencias digitales. Tomando las respuestas a título personal se buscó su opinión sobre los siguientes aspectos:

- Después de la crisis, las competencias digitales serán más importantes en el mercado laboral: 92,5\% de respuestas positivas.

- Intención de mejorar las habilidades y competencias digitales en el futuro: 74,9\%.

- Planear tomar nuevas iniciativas / cursos / capacitación para mejorar sus habilidades y competencias digitales en el futuro:

o $88 \%$ para educadores

o $\quad 83,5 \%$ para personal de educación y formación

o por el contrario, un 43,5\% de padres no tienen pensado realizar acciones de futuro en el ámbito digital. 
- Entre los tipos de habilidades y competencias digitales que les gustaría mejorar en el futuro, destaca la creación de contenidos digitales: 60 \% en educadores; $46 \%$ en personal de educación y formación.

b) Puntos de vista sobre el papel de la educación digital en el futuro. Del total de la muestra, un 94,5\% consideraban que la pandemia y los cambios que supusieron en las modalidades de enseñanza y aprendizaje tendría un impacto a largo plazo en los procesos formativos. Se propuso a los educadores y personal de educación que señalaran las principales ventajas de la educación digital para el futuro, así como las desventajas más destacables. (Tabla 5). También se les pidió que indicaran los beneficios potenciales y las barreras más destacables del modelo de aprendizaje mixto (combinación de aprendizaje presencial y en línea), de gran desarrollo en la fase de la pandemia que estamos viviendo en la actualidad (Tabla 6).

Tabla 5 .

La educación digital en el futuro.

\begin{tabular}{llll}
\hline Principales ventajas & & Principales desventajas & \\
\hline $\begin{array}{l}\text { Flexibilidad, enseñanza y aprendizaje } \\
\text { a su propio ritmo }\end{array}$ & $64,3 \%$ & $\begin{array}{l}\text { Menos interacción comunicación } \\
\text { cara a cara }\end{array}$ & $60,4 \%$ \\
$\begin{array}{l}\text { Formas innovadoras y atractivas de } \\
\text { enseñanza y aprendizaje }\end{array}$ & $54,6 \%$ & $\begin{array}{l}\text { Necesidad de conectividad y } \\
\text { equipamiento adecuado }\end{array}$ & $37,5 \%$ \\
$\begin{array}{l}\text { Ayudar a los alumnos a desarrollar } \\
\begin{array}{l}\text { sus habilidades y competencias } \\
\text { digitales }\end{array}\end{array}$ & Incapacidad para hacer prácticas & \\
\hline
\end{tabular}

Fuente: elaboración propia a partir de Plan de Acción Digital 2021-2027

Tabla 6.

Combinación del aprendizaje presencial y en línea.

\begin{tabular}{|c|c|c|c|}
\hline \multicolumn{2}{|l|}{ Beneficios potenciales } & \multicolumn{2}{|l|}{ Posibles barreras } \\
\hline Más flexibilidad, aprendiendo a su & $68,4 \%$ & Los estudiantes sin acceso a & $73,9 \%$ \\
\hline propio ritmo & $62.1 \%$ & $\begin{array}{l}\text { tecnoiogias digitales adecuadas } \\
\text { están excluidos }\end{array}$ & $69,6 \%$ \\
\hline $\begin{array}{l}\text { Comunicación cara a cara e } \\
\text { interacción entre estudiantes y } \\
\text { educadores }\end{array}$ & $59,2 \%$ & $\begin{array}{l}\text { Mayor carga de trabajo para el } \\
\text { personal de formación }\end{array}$ & $67,4 \%$ \\
\hline $\begin{array}{l}\text { Oportunidad apoyar estudiantes de } \\
\text { grupos desfavorecidos }\end{array}$ & & $\begin{array}{l}\text { Dificultad para los padres para } \\
\text { combinar trabajo y educación }\end{array}$ & \\
\hline
\end{tabular}

Fuente: elaboración propia a partir de Plan de Acción Digital 2021-2027

c) Puntos de vista sobre cómo la Unión Europea podría agregar valor cuando se trata de educación digital. Este apartado logra establecer un consenso entre todos los participantes (instituciones, organizaciones, padres y educadores) en cuanto que los esfuerzos de la UE en lo referente a la educación digital se deben centrar en la formación y orientación de los profesores, con un 58,7\%. 


\section{Discusión y conclusiones}

Tras el primer confinamiento, la vuelta a las rutinas educativas no ha llegado a recuperar todos los hábitos previos, generándose un vocabulario ya asimilado: bimodalidad, sincrónico, online, que conforma un presente difícil de desarrollar sin la contribución de las tecnologías digitales. El ámbito educativo se ha visto afectado de manera notable si partimos de unos centros formativos que permanecieron cerrados durante varios meses, poniendo en jaque la necesidad de continuar con los procesos educativos; condicionando los aprendizajes a la intensa colaboración de las familias; dificultando los procesos evaluativos al no contar con los registros de las pruebas pertinentes. La realidad surgida tras el confinamiento también ha mostrado notables diferencias, revelándose como un verdadero reto la obligación de mantener la distancia social en un área caracterizada por la gran densidad poblacional. A todo esto, el rol del profesorado ha adquirido una nueva dimensión, exigida por la dificultad de llevar a cabo los procesos de enseñanza y aprendizaje. Por lo que la triangulación desarrollo profesional docente, rendimiento de los estudiantes y contexto escolar sin duda van a marcar las reformas a introducir (Fischer et al., 2018), representando la gestión de estas variables todo un desafío. Eso sí, los cambios a implementar tendrán que ocupar todos y cada uno de los diferentes niveles de los sistemas educativos, a saber, políticos, institucionales e individuales (Bozkurt et al., 2020); y tendrán que contar con la presencia de la tecnología digital.

La pandemia COVID-19 más que presentar nuevos desafíos metodológicos ha evidenciado las carencias de las que adolece un sistema que lleva hablando de las nuevas tecnologías varias décadas, pero sobre quien ha caído el peso de la inadaptación con severa rotundidad. El análisis de datos que hemos realizado referidos a la formación del profesorado en TIC antes y durante la pandemia nos conducen a valorar la dificultad que implica acompañar al alumnado en sus procesos de aprendizaje si el profesorado queda anclado en prácticas no evolucionadas (Paniagua e Istance, 2018). Sin embargo, la renovación del profesorado depende en gran medida de unas instituciones motivadoras y facilitadoras (Tejada y Pozos, 2018), pues de lo contrario volvemos a dejar la calidad de la respuesta en la individualidad del docente.

En el año 2018, el 39 \% del profesorado de la UE consideraba tener unos niveles de capacitación buenos o muy buenos en el área de las TIC; durante la pandemia, más del 88 \% reflejaban la necesidad de una mayor formación digital; dato este último que casi duplica a lo manifestado por las familias. Respondiendo a la primera de las cuestiones que nos planteábamos, parece claro que la percepción de los profesores sobre su propia capacitación en TIC se ha modificado, pues en el momento del preconfinamiento se había producido un estancamiento en la evolución de la percepción de la necesidad de formación en esta área, donde solo un $15 \%$ de los profesores consideraban que una formación específica en TIC fuera fundamental. En esta línea, un estudio del año 2017 con 980 respuestas de profesores universitarios de 25 universidades españolas ponía de relieve la contradicción que suponía la elevada oferta formativa en modelos de enseñanza online o bimodal y su escasa presencia en la cultura de los docentes (Espejo, Lázaro y Álvarez, 2020).

Sin embargo, la experiencia del confinamiento y la obligación de llevar a cabo un desempeño de la enseñanza mayoritariamente online durante los primeros meses, y en modalidad de semipresencialidad posteriormente, el 74,5\% del profesorado expresaba su intención de mejorar sus habilidades y competencias digitales, sobre todo en cuanto a 
la creación de contenidos educativos en formato digital. Una nueva cultura educativa que sin duda se verá reforzada al establecerse lo que Casillas, Cabezas, Ibarra y Rodríguez (2020) establecieron como la relación positiva entre la variable de manejo de dispositivos y la actitud favorable hacia las TIC.

En cuanto a la segunda cuestión que abordamos en esta investigación y que hace referencia a las ventajas e inconvenientes que los docentes aprecian en la enseñanza online o semipresencial, el profesorado subraya como principal desventaja la brecha digital que se puede dar en una parte del alumnado, al no contar con los medios técnicos necesarios. Por lo que proponen, como forma de subsanar este déficit, la necesidad de invertir tanto en equipos para los estudiantes como en formación del profesorado en educación digital. Es el problema de la brecha digital una realidad que no se puede obviar. Si bien la curva es descendente, en el año 2009 un $15 \%$ de los estudiantes de los países miembros de la OCDE afirmaban no disponer de acceso a internet, para reducirse a un $5 \%$ nueve años más tarde (OCDE, 2019) y el acceso a internet en los hogares de la Unión Europea había aumentado 29 puntos porcentuales entre los años 2008 y 2018, alcanzando el $89 \%$, continúa siendo una de las preocupaciones del profesorado, considerando esta desigualdad como la principal barrera en la metodología de aprendizaje en línea, presencial o mixta.

Es evidente que las herramientas digitales propondrán ventajas, facilitando el desarrollo profesional docente (Casillas, Cabezas, Ibarra y Rodríguez, 2020) y conllevarán riesgos, sobre todo cuando el uso se convierta en abuso (González, Córdoba y Gómez, 2020); pero, ante todo, demandará una planificación que permita anticipar los futuros escenarios en los que los centros educativos se verán inmersos. Si bien la actual dependencia de lo digital en la actividad docente no constituye el marco teórico sobre el que se perfilarán las próximas estructuras educativas, no cabe duda de que ha supuesto toda una modificación con respecto a su valoración por parte del profesorado (Porlán, 2020).

$\mathrm{Al}$ abordar el último de los ámbitos tratados por la Consulta Pública Abierta del año 2020, todos los agentes educativos y el propio alumnado coinciden en que la Unión Europea debe centrar sus esfuerzos en la formación y orientación del profesorado en el área digital. En este sentido, desde el programa Erasmus+ se lanzaron dos convocatorias en agosto del 2020 para fomentar la puesta en marcha de proyectos, para todas las etapas educativas, con el objetivo de mejorar la educación online y para apoyar la capacitación digital de los docentes.

Las diferentes líneas de trabajo que se nos presentan tras el análisis de la cuestión tendrán que orientarse hacia una necesidad que surge desde el momento en el que lo digital se incorpora al espacio educativo, a saber, conseguir trasladar la competencia digital docente en propuestas de aula, (Gisbert, González, y Esteve, 2016). Propuestas para unas aulas que, con independencia de si son virtuales o físicas, favorezcan la identificación del alumnado con su tarea, con sus compañeros y con el profesorado. Es decir, superar la alfabetización digital para elaborarla como identidad digital, en su dimensión comunicativa y creativa (Sanabria y Cepeda, 2016); y vencer la desconexión social que acompaña los procesos de enseñanza y aprendizaje en los contextos online (Sangrà, 2020). Alcanzar, por tanto, un nivel de comunicación educativa en el que ante situaciones excepcionales la distancia no elimine la expresión de sentimientos, emociones e ideas. 


\section{Referencias}

Bauman, Z. (2008). Los retos de la educación en la modernidad líquida. Barcelona: Gedisa.

Bozkurt et al. (2020). A global outlook to the interruption of education due to COVID-19 Pandemic: Navigating in a time of uncertainty and crisis. Asian Journal of Distance Education, 15,(1), 1-126.

Cabero, J. y Valencia, R. (2019). TIC para la inclusión: una mirada desde Latinoamérica. Aula Abierta, 48(2), 139-146. doi: https://doi.org/o.17811/rifie.48.2.2019.139-146.

Casillas, S., Cabezas, M., Ibarra, M. y Rodríguez, G. (2020). El Profesorado Universitario en la Sociedad del Conocimiento: manejo y actitud hacia las TIC. Bordón. Revista de Pedagogía, 72(3), 45-63. doi: doi.org/10.13042/Bordon.2020.76746

CEDEFOP. (2020). Digital gap during COVID-19 for VET learners at risk in Europe Recuperado de https://www.cedefop.europa.eu/files/digital_gap_during_ covid-19.pdf

Comisión Europea. (2017). Marco Europeo para la competencia digital del profesorado (DigCompEdu). Recuperado de https://ec.europa.eu/jrc/sites/jrcsh/files/ digcompedu_leaflet_es-nov2017pdf.pdf

Comisión Europea (2020a). Digital Education Action Plan (2021-2027). Communication. Recuperado de https://ec.europa.eu/education/sites/education/files/documentlibrary-docs/deap-communication-sept2020_en.pdf

Comisión Europea. (2020b). Public consultation launched on the new Digital Education Action Plan. Recuperado de https://ec.europa.eu/education/news/publicconsultation-new-digital-education-action-plan_es

Core, P. (2012). ¿Resistir o innovar? La figura del docente entre crisis y relanzamiento de la formación. En J.C. Torre (Coord.), Educación y nuevas sociedades. Madrid: Universidad P. Comillas, ACISE-FIUC.

Consejo Europeo (2006). Recomendación del Parlamento Europeo y del Consejo, de 18 de diciembre de 2006, sobre las competencias clave para el aprendizaje permanente. (DO, 394/10, 2006).

Consejo Europeo (2018). Recomendación del Consejo de 22 de mayo de 2018, relativa a las competencias clave para el aprendizaje permanente. (DO, C 189/1, 2018).

Egido, I. (2011). Dilemas y cambios en la formación de maestros: 50 años de la historia de España en perspectiva europea. Tendencias pedagógicas 18, 33-50.

Espejo B., Lázaro L. y Álvarez, G. (2020). Estudio del blended learning en el gobierno universitario español: tecnocracia digital versus conocimiento científico. Bordón $72(3),, 85-103$.

Gisbert, M., González, J. y Esteve, F. M. (2016). Competencia digital y competencia digital docente: una panorámica sobre el estado de la cuestión. Revista Interuniversitaria de Investigación en Tecnología Educativa. https://doi. org/10.6018/riite2016/257631 
Fernández Enguita, M. (2020). Una profesión firme para un contexto inestable. Temas para el debate, 309, 25-28.

Fernández Prieto, M. S. (2015). La aplicación de las nuevas tecnologías en la educación. Tendencias Pedagógicas, 6, 139-148. Recuperado de https://revistas.uam.es/ tendenciaspedagogicas/article/view/1811

Fischer, C. et al. (2018). Investigating relationships between school context, teacher professional development, teaching practices, and student achievement in response to a nationwide science reform. Teaching and Teacher Education, 72, 107-121.

Garcia Aretio, L. (2019). Necesidad de una educación digital en un mundo digital. RIED. Revista Iberoamericana de Educación a Distancia, 22(2), pp. 09-22. doi: http:// dx.doi.org/10.5944/ried.22.2.23911

González, E., Córdoba, A y Gómez, M. (2020). Una semana sin smartphone: usos, abuso y dependencia del teléfono móvil en jóvenes. Bordón. Revista de Pedagogía, 72(3). doi:doi.org/10.13042/Bordon.2020.79296

Lago, S. (Coord.) (2019). Políticas públicas e inclusión digital. Un recorrido por los núcleos de acceso al conocimiento. Buenos Aires: Instituto de Investigaciones Gino Germani.

MEFP. (2019). Estudio Internacional de Enseñanza y Aprendizaje, TALIS 2018, informe español. Madrid: Ministerio de Educación y Formación Profesional.

Mora, J. G. (2011). Formando en competencias: ¿Un nuevo paradigma? Fundación Conocimiento y Desarrollo (CYD).

OECD. (1989). Information Technologies in Education: The Quest for Quality Software. Paris: Organization for Economic Cooperation and Development.

Olarte, S. (2017). Brecha digital, pobreza y exclusión social. Revista andaluza de trabajo y bienestar social, 138, 285-313.

Opfer, V. D. \& Pedder, D., 2011. Conceptualizing Teacher Professional Learning. Review of Educational Research, 81(3), 376-407.

Paniagua, A. \& Istance, D. (2018). Teachers as Designers of Learning Environments: The Importance of Innovative Pedagogies, Educational Research and Innovation. París: OECD. doi: doi.org/10.1787/9789264085374-en.

Pegalajar, M. C. (2017). El futuro docente ante el uso de las TIC para la educación inclusiva. Digital Education Review, 31, 131-148. Recuperado de http://digibug. ugr.es/bitstream/handle/10481/39371/Vol6\%281\%29_016_jett_pegalajar. pdf? sequence $=1 \&$ isAllowed $=\mathrm{y}$

Porlán, R. (2020). El cambio de la enseñanza y el aprendizaje en tiempos de pandemia. Revista de Educación Ambiental y Sostenibilidad 2(1), 1502. doi: 10.25267/Rev_ educ_ambient_sostenibilidad.2020.v2.i1.1502 
Rodríguez, M. y Arroyo, M. J. (2014). Las TIC al servicio de la inclusión educativa. Digital Education Review, 25, 108-126. Recuperado de http://revistes.ub.edu/index.php/ der/article/viewFile/11331/pdf

Ribes, E. (2011). El concepto de competencia: su pertinencia en el desarrollo psicológico y la educación. Bordón, 63(1), 33-45.

Ribés, A. S., Moliner, L. y Alegre, F. J. (2020). Educación inclusiva y tic: un análisis de las percepciones y prácticas docentes. Bordón 72(3), 123-138. doi: 10.13042/ Bordon.2020.69852

Ruiz, C. (2010). La Educación en la sociedad postmoderna: Desafíos y oportunidades. Revista Complutense de Educación, 21(1), 173-188.

Sanabria, A. L. y Cepeda, O. (2016). La educación para la competencia digital en los centros escolares: la ciudadanía digital. Revista Latinoamericana de Tecnología Educativa, 15(2), 95-112.

Sangrà, A. (coord.) (2020). Decálogo para la mejora de la docencia online. Propuestas para educar en contextos presenciales discontinuos. Barcelona: Editorial UOC.

Schwab, K., y Davis, N. (2018). Shaping the Future of the Fourth Industrial Revolution. London: Penguin Books.

Tejada, J. y Pozos, K. V. (2018). Nuevos escenarios y competencias digitales docentes: hacia la profesionalización docente con TIC. Profesorado, Revista de Currículum y Formación del Profesorado, 22(1), 25-51. doi: doi.org/10.30827/recfpro. v22i1.63620

Tobon, S. (2020). Educación en línea y socioformación. En J. Luna-Nemecio (Coord.), Memorias del Quinto Congreso Internacional de Investigación en Socioformación y Sociedad del Conocimiento.

Torrecillas, C. (2020). El reto de la docencia online para las universidades públicas españolas ante la pandemia del Covid-19. ICEI Papers COVID-19, (16). Recuperado de: https://eprints.ucm.es/60050/

Tumino, M. C. y Bournissen, J. M. (2019). Integración de las TIC en el aula e impacto en los estudiantes: elaboración y validación de escalas. International Journal of Educational Research and Innovation (IJERI), 13, 62-73.

UNESCO. (1995) Documento de Política para el Cambio y el Desarrollo en la Educación Superior. París: UNESCO.

UNESCO. (1999). Los docentes, la enseñanza y las nuevas tecnologías. En Informe mundial sobre educación. Madrid, Santillana/UNESCO.

UNESCO. (2008). Estándares de competencia en TIC para docentes. Paris: UNESCO. 